\title{
Design an Intelligent IoT system for terrain road haulages to optimize load with transmission and bottleneck control
}

\author{
${ }^{1}$ Ashiq MI ${ }^{1},{ }^{2}$ Shivani Sethi, ${ }^{3}$ Gregory Nannam, ${ }^{4}$ Mohammed Niyas Dubaskaja, ${ }^{5}$ Vijayakumar Sampathkumar \\ ${ }^{1}$ Functional Lead, Cognizant Technology Solutions, India \\ ${ }^{2}$ Manager Projects, Cognizant Technology Solutions, India \\ ${ }^{3,4,5}$ Functional Architect, Cognizant Technology Solutions, India
}

\begin{abstract}
The movement of goods and services by road is the only mode of transport in high altitude terrain regions. Most of the large scale transportation is done by trucks and any kind of breakdown, accidents, over loading, reckless driving and not giving way for uphill vehicles results in traffic bottleneck and fatal aftermath. During monsoons the transportation is either halted or more challenging amidst rain, fog, landslides and deteriorated roads. The objective is to design an AI integrated IoT cloud mobile platform to ensure a safe and controlled driving environment.
\end{abstract}

Keywords - IoT - Internet of Things, AI - Artificial Intelligence, HGV - Heavy Goods Vehicle, GPS - Global Positioning System,

\section{INTRODUCTION}

$\mathrm{R}$ oad traffic accidents are responsible for 1.2 million deaths worldwide each year and will become the 3rd largest contributor to the global burden of diseases after ischemic heart diseases and depression. [1] Ghat roads are access routes into the mountains with number of hairpin bends, which is very risky as compare to normal routes. So chances of accidents in ghat section is more because of narrow road width, sharp bends, improper camber and valley side. While driving on roads at Ghats section many drivers faces accidents which result them into serious injuries or even death. The main reason behind this accidents is curves and bends of road while turning in Ghats. It becomes difficult to see vehicle coming from other lane at turning. If two vehicles comes face to face while turning it creates a chance of accidents and it becomes difficult to handle. [2] Road users see it as a major cause of concern as it affects their travel plans. However, time restriction on lorry movement failed to yield any results as overloaded vehicles continue to develop snag while negotiating the hair bends resulting in traffic congestion.

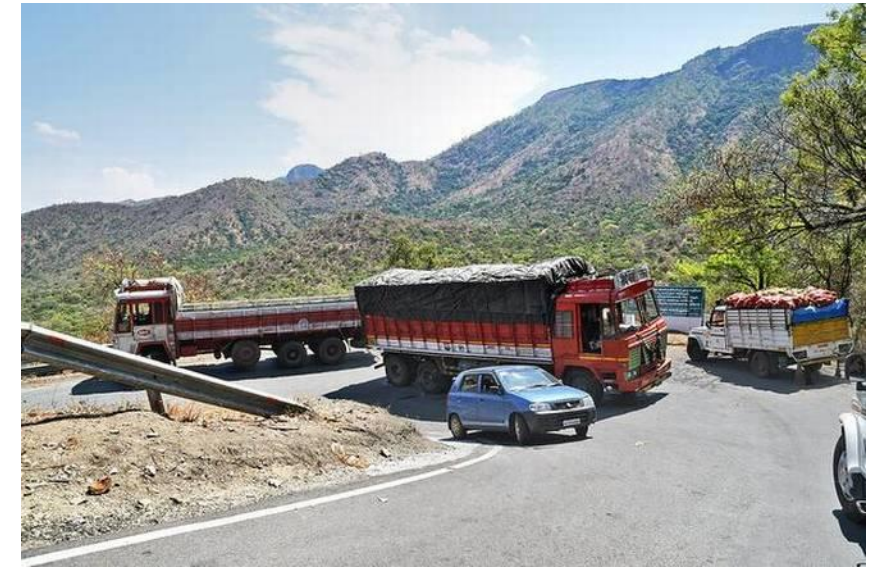

Fig.1.1 Lorry breakdown at hairpin bend on Dhimbam Ghat

The issue could be solved only if restriction was imposed on height and weight for loaded lorries. [3] Recently, there have been a significant number of vehicles illegally overloaded and the damage vehicles cause on the road is in direct proportion to the axle weight by 4th power. The overloaded transportation would greatly increase the cost for the pavement maintenance and repair, shorten the service life of pavement, even affect the traffic safety and capability. So it is imperative to build a weigh station to solve these problems. Traditionally the weights of vehicles were measured and collected by placing it on the scale while the vehicle is at rest. [4] Driver Alert System is basically designed to avoid road accidents in hilly areas by alerting the drivers of opposite ends against arrival of each other. After alert signal like light glows driver will slow down its speed thus avoiding quashing with each other. Programmable logic controllers are utilized and programmed to get the desired output via sensors. [5] The implementation of IoT is continuously increasing in the automotive sector. By 2023, automakers will sell more than $\$ 73$ million connected vehicles across the global customer base. It is expected that by the end of 2025, all the newly manufactured cars will be connected with the IoT. The current state and future predictions about IoT in the automobile industry clearly show the adoption of IoT is rising rapidly in fact, it is increasing exponentially. IoT can facilitate secure 
and seamless communication between multiple vehicles by developing a common Vehicle to Vehicle network. This can especially be beneficial in the prevention of road accidents by allowing the vehicles to share their real-time data about speed, location, and route with other cars. The sensors embedded in the vehicles can generate real-time alerts and send notifications to the driver or the manager of the vehicle in case of an emergency. These real-time alerts help them take timely actions to prevent any mishappening, thereby making realtime monitoring one of the most important features of automotive IoT. The trucks are now tooled up with smart features like weight estimation, real-time position tracking, and many others. IoT is benefitting the fleet industry tremendously with capabilities like real-time location monitoring, route management, fuel \& mileage monitoring, and condition monitoring. The sensors embedded in the fleet collect, analyze, and visualize data on the central dashboards from where the fleet operators can get real-time information for effective decision-making. [6] Road transport is one of the sectors where AI has most successfully been applied, opening up entirely new levels of cooperation between various road users. Worldwide, automotive manufacturers, technology firms and research groups are exploring AI technologies to develop automated vehicles for use in commercial as well as personal transportation. Such vehicles are based on a variety of sensors (such as GPS, cameras, radar), in combination with actuators (devices which transform an input signal into motion), control units and software. Some of these technologies only take over certain driving functions (like parking), others are intended to completely replace the human driver. AI technologies that take over certain driving functions are already widely available on the European Union market, while fully automated vehicles are being tested (including to deliver parcels) in a limited number of driving situations and areas.

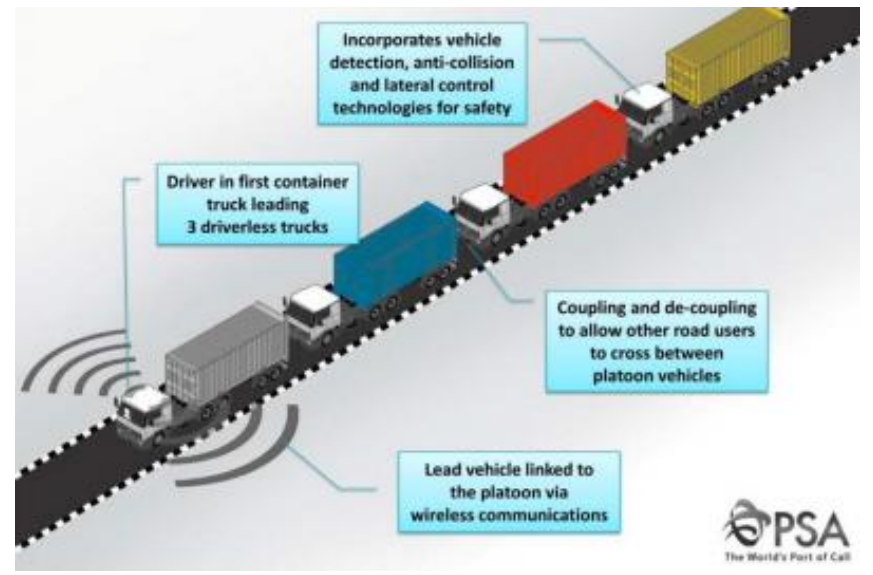

Fig.1.2 Illustration of truck platooning

AI also makes truck platooning possible - the coupling of several HGV within minimal distance of each other, allowing them to automatically and simultaneously accelerate or brake. While the lead HGV is driven by a human driver, drivers in those following may only be present in case complex traffic situations (such as roundabouts) or unexpected incidents arise, rather than actively driving. In the future, it is expected that the responsibilities of the drivers of following HGVs will reduce progressively, until ultimately they are no longer necessary. Although HGV manufacturers are already testing truck platooning in several European Union countries, further tests are still needed on multi-brand truck platooning in more complex traffic situations, to verify safety requirements are met. [7] Driver app companies, such as 99 and Samsara, have developed a facial recognition tool that has its purpose to increase even more the safety of the driving experience. The 99 system, developed by engineers and programmers in three countries - Brazil, USA and China - automatically identifies the face of all drivers before they even connect to the app. Furthermore, the technology will be applied periodically in every driver registered in the app, including those who already passed by an identity check. 99 performs the procedure through a partnership with the Department of Motor Vehicles of each region they are performing, where the collected image in the facial reader is automatically validated with the saved photo in the data bank. Many facial recognition programs also perform along with AI, and can identify the driver use patterns and request additional checking, if necessary. The company informs that random verifications will also be performed. Besides bringing more safety for not just the driver but also all other people on the road, applying one more confirmation step also has the goal to protect the driver's identity. The safety of everyone on the road is the main priority. Facial recognition now joins to several tools of protection of the users, such as dash cameras and vehicle emergency buttons. Periodically, the app asks the drivers to do a facial recognition, anytime of the day. The drivers should put their faces in the circle and wait a few seconds while the verification is performed. In case it is approved, the driver can normally drive. If not, they will be able to request a review from the app. Inside the vehicle, with the aid of a camera and biometric sensors, artificial intelligence monitors the driver behavior and modify the configuration of the cabin, such as heat, light and media. AI recognition systems can go as far as detecting if the driver is bored and play their podcast or favorite song automatically. And if the driver is starting to fall asleep while driving, AI will decrease the vehicle temperature to make sure someone is aware. So, with time, the technology will be able to learn personal preferences of the user and request personalized responses when necessary. It's a big leap in technology for the trucking industry, and one that must be fully studied and then slowly adopted so that all drivers have a full understanding of the impacts of AI. [8] IoT and fleet management systems company Samsara launched new artificial intelligence (AI) features for its cameras to help fleets monitor for distracted driving and tailgating. The features use video recognition tech to keep an eye on drivers and the road, automatically alerting the fleet manager if problems arise. Samsara's approach in this case uses a facial recognition system with a camera facing the driver. That can detect things like nodding off or looking down away from the road, perhaps at a smartphone. Then fold in a forward-facing camera view as well that can recognize 
other vehicles, road signs and so on. [9] In its most basic form, facial recognition uses cameras to identify a person via their facial features, placement of the eyes, nose, mouth and possibly any other distinguishing features like the hairline. Camera systems that recognize specific objects were only found in manufacturing up until a few years ago, that was due to the fact they required so much power to feed the hungry processors that churned the data which figured out what each picture was. With the advent of smaller, highly optimized computing chips, image and facial recognition can now be built into pretty much anything. There are numerous systems on the market, either using two dimensional or three dimensional camera setup. Most of the two dimensional units use two cameras mounted in the dash to give a stereoscopic view of the world in front of them.

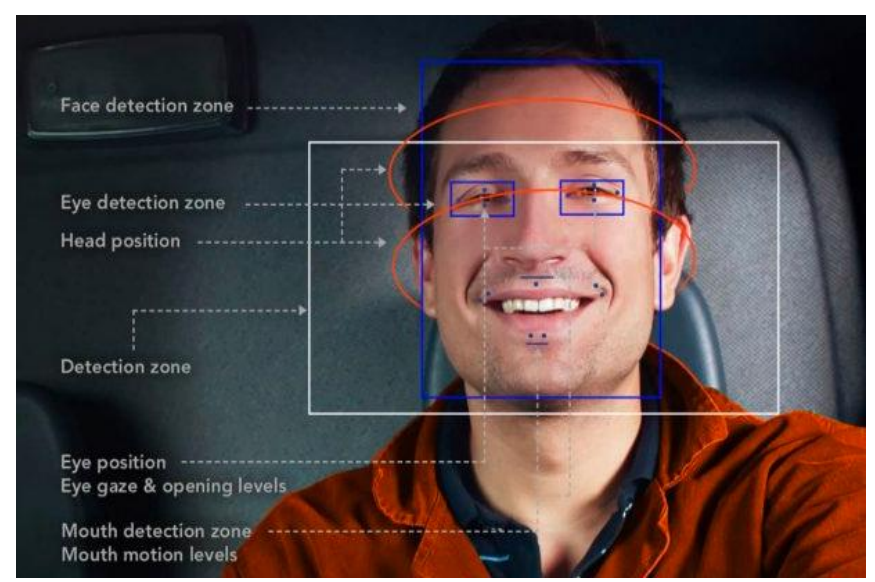

Fig 1.3 Facial detection zones

The three dimensional systems can utilize a single camera. There are also newer, more modern systems that rely on infrared camera which can see better in low light and night scenarios. The cameras capture the scene in the form of images multiple times a second, these are fed to a microprocessor that makes sense of the image by picking apart various data points. If the drivers head has started to tilt, their eye line isn't straight ahead, maybe their blink rate has increased, or the redness in their eyes is becoming more intense. They can also track body and head position within the car and even how many occupants are in the vehicle. From here the system will decide if any intervention is needed, this could be in the form of an audible warning or a light, a vibration of the seat or steering wheel and possibly even a slight take-over of the vehicle controls - slowing the car down or moving lane to a lower speed. Looking after a fleet of drivers can be hard work, you need to know where your drivers are at all times, how long they've been driving and if they're safe out on the roads. This is where facial recognition steps in, not only can you monitor where a driver is looking but you can also keep an eye on their emotions, from surprise, to fear and even anger. This can be used to determine how a particular driver is feeling, are they happy in their work, and are they comfortable. Maybe they're easily frustrated and need to calm down when out on the road, or are they spending too much time distracted making phone calls.

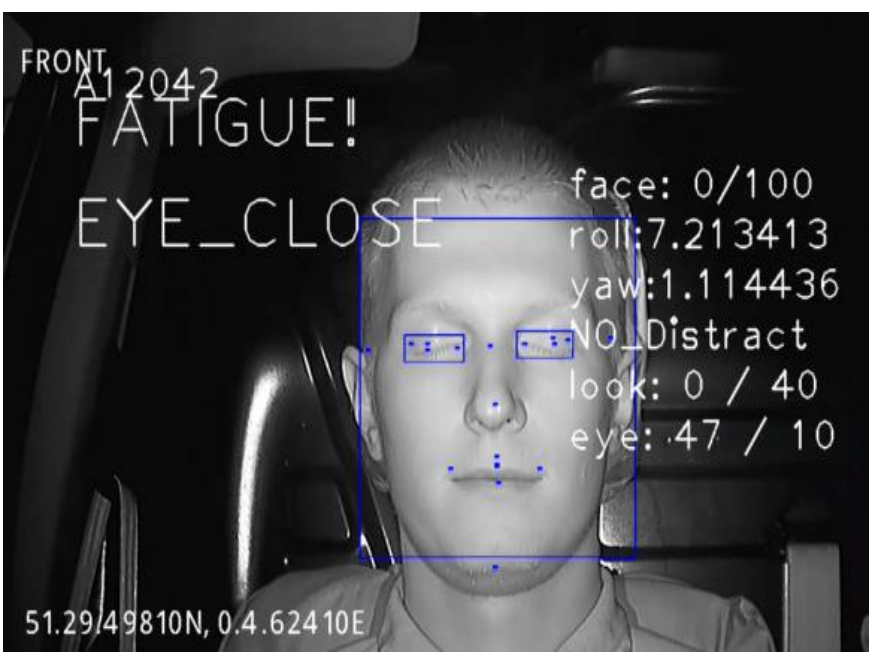

Fig 1.4 Facial Recognition - signs of fatigue

Deeply embedding what a facial recognition system sees with other vehicle safety systems could lead to even greater crash prevention measures. If you're eyes leave the straight ahead position for more than a few seconds the car could automatically start braking, priming the brake cylinder and tightening the seatbelts in case of an accident. If these systems become prominent, the guardian angel effect of having current systems like active city braking and pre-collision detection would increase exponentially. [10] Moqups makes it easy for remote teams to create quick wireframes and collaborate online in real time. With our easy-to-use wireframe tool, your team can design user interfaces for webpages and mobile apps. Simplify your design process and move seamlessly from low-fidelity to high-fidelity. Designers and non-designers alike can drag and drop pre-made UI elements for fast, convenient wire framing. Work out ideas, solve problems, and come to a quick consensus. Connect to Jira, Confluence, Slack, Trello, Dropbox and Google Drive to create a fullyintegrated workflow. Your team can share, communicate, and coordinate - at every level - with our collaborative wire framing tool. [11]

\section{PROBLEM DESCRIPTION}

The passengers and vehicles travelling on Ghat section experience traffic bottleneck and life threatening accidents due to technical and mechanical snag developed by heavy goods vehicles. The evident cause to this snag is low vehicle maintenance, poor driving etiquettes, environmental factors, not adhering to traffic and motor vehicle rules. The existing system lacks an official cloud application integrated with live sensors for an automated approval process. Thus enabling only sturdy trucks with disciplined drivers to ply through the Ghat section. 


\section{PROPOSED ARCHITECTURE}

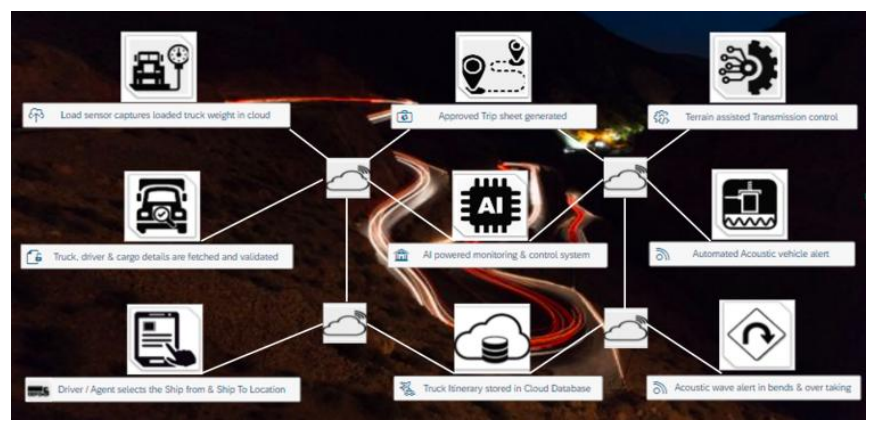

Fig 3.1 Architectural Design

An attempt has been taken to propose a mobile application based registration, approval and monitoring system for heavy goods vehicles plying through the Ghat sections. The mobile application is connected through cloud with the live sensors installed in the truck. The mobile application features sensor calibration only for authorized personnel to maintain data accuracy. Approval decisions, alert and control mechanisms will be performed by AI. This application will guide and monitor the truck driver from registration, loading, trip start, unloading and trip end thereby avoiding traffic congestions and accident control.

\subsection{Prerequisite for the proposed architecture:}

1. User has registered and active in the new mobile application

2. Installation and functioning of sensors in trucks

3. Live sensors connected to mobile application and cloud database

4. Mobile front camera mounted on truck dashboard and facing the driver

5. Mobile connected to the vehicle ignition and drive train system

\subsection{Mobile Application onboarding flow:}

The mobile application prototype and user navigations are represented as a mobile application wireframes in Fig 3.2.

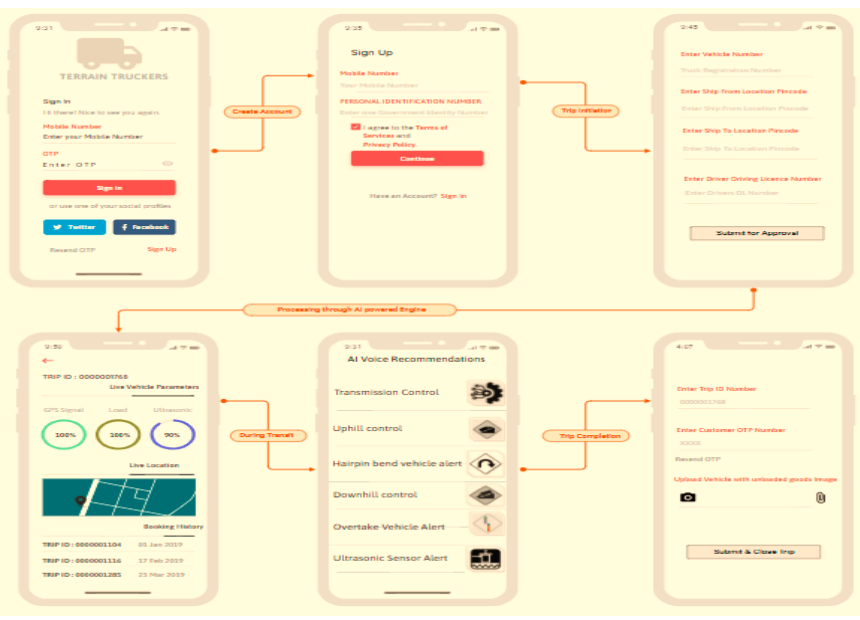

Fig 3.2 Mobile Application Wireframes
The proposed mobile application onboarding steps integrating sensors, cloud database and AI are presented below,

\subsubsection{User Registration:}

The driver / agent needs to register through the new mobile application with their mobile number and personal identification number.

\subsubsection{Trip Registration:}

The vehicle registration number, driver details, ship from, ship to location and driving licence number are keyed in for the trip registration.

\subsubsection{Heavy Goods Vehicle Approval:}

The vehicle history details like year of manufacture, fitness, insurance, permit, turning radius, driver experience and accident history will be fetched and compared with the itinerary details like bends, narrow bridges and sharp turns. The vehicle and driver details are verified and digitally approved with the government records to avoid vehicle audit in the check post and toll plazas.

\subsubsection{Load Approval:}

The load approval will be provided only for a certain tonnage considering the maximum threshold limit of the vehicle and the terrain government norms. The load sensors provide a live update from the time of loading the vehicle, thus reducing the travel time to weigh bridges and reducing illegal loading of goods beyond the threshold limit.

\subsubsection{Database Update:}

The vehicle and load details will be validated and verified using the AI powered cloud platform. On successful verification, the vehicle details will be captured in cloud database. Variations of any kind can be live tracked by the customer, government officials and truck owners.

\subsubsection{Acoustic Alert system:}

The trucks enabled with acoustic sensors send a specific frequency that differs during uphill and downhill which can be received by other vehicles. This signal triggers an alert to other vehicles internally via sound alert tone to stop during hairpin bends and pass.

\subsubsection{Drive Train Control system:}

The drive train control sensor automatically locks the higher gear in trucks when travelling downhill and releases when the trucks reaches the linear surface and also based on GPS location.

\subsubsection{Face Detection system:}

To curb others from driving the truck, the image of the driver captured in cloud, will be monitored through the face detection software randomly during the trip and any variations, fatigue, undesirable emotions will bring the vehicle to a halt position and trigger an alert to the truck owner. 


\subsubsection{Unload Approval:}

The goods post reaching the customer premises is unloaded. One time password will be triggered to the customer and the same needs to be entered in the proposed driver's mobile application followed by uploading of a combined picture of unloaded goods, truck and the driver from the customer location. This improves the customer satisfaction and driver reliability. The trip will be updated as complete in the database, post unload approval.

\section{CONCLUSION}

This paper proposes an architecture design to build an AI and IoT integrated mobile application enabling a better user experience platform for the transportation line of business. Proposed solution will address the pitfall in the existing process like starting a trip without prior approval and vehicle fitness, restrict helpers and cleaners drive the truck leading to accidents and traffic bottlenecks. The truck and driver history is stored in a centralized cloud database making it easy during inter and intra state vehicle transport audits. This system also provides vehicle based ultrasonic automated alert technique and virtual monitoring that will serve as a guardian angel to save millions by ensuring a safe, controlled and disciplined driving.

\section{REFERENCES}

[1] Joshi AK, Joshi C, Singh M, Singh V, (2014), "Road traffic accidents in hilly regions of northern India: What has to be done?"World Journal of Emergency Medicine, Vol.5, No.2. pp.112-115.

[2] Harshada Targe et al, (2018),"Advance Road Safety For Ghat Road's At Hairpin Bend" International Research Journal of Engineering and Technology (IRJET),Vol.05.Issue.1.pp.251-253

[3] https://www.thehindu.com/news/cities/Coimbatore/frequentbreakdown-of-lorries-on-dhimbam-ghat-road-worries-roadusers/article26900190.ece

[4] Mehran Safdar, (2015), "A Mobile Vehicle Weight Sensor and its Application in Transportation", 1st International Electronic Conference on Remote Sensing

[5] Bishwajeet Singh, Mohit Arvind, Divya Asija, Pallavi Choudekar, Ruchira, (2019),"Driver Alert System for Hilly Roads at Hair Pin Bends" International Research Journal of Engineering and Technology (IRJET),Vol.06.Issue.9S.pp.85-90

[6] https://www.biz4intellia.com/blog/how-iot-technology-issupervising-operations-in-automotive-industry/

[7] https://www.europarl.europa.eu/RegData/etudes/BRIE/2019/6356 09/EPRS_BRI(2019)635609_EN.pdf

[8] https://unitedworldtransportation.com/how-facial-recognition-andai-is-improving-the-safety-of-truck-drivers/

[9] https://www.fleetowner.com/safety/article/21702474/samsaradelves-into-ai-fleet-safety-upgrades-camera-features

[10] https://www.exeros-technologies.com/how-facial-recognitiontechnology-can-help-prevent-truck-driver-fatigue/

[11] https://app.moqups.com/ 